\title{
In this issue
}

By John Ashkenas, Science Editor

Building bulkier smooth muscle cells through endoreduplication

\section{(See article on pages 1011-1020)}

In normal aging, unusually large smooth muscle cells (SMCs) accumulate in the walls of the aorta and other socalled capacitance arteries, but not in other arteries or veins. Interestingly, these cells appear to have undergone endoreduplication, a round of chromosomal duplication without the usual cell division that leaves the cell with twice the usual complement of DNA. Such tetraploid cells can also be generated in cultured cells using microtubuledepolymerizing drugs, which disrupt the mitotic spindle. Tetraploid cells reach large sizes, probably because they extend their cell cycles at the G1 phase, a period when cells accumulate mass. Higher ploidy cells are observed at some developmental stages or cell lineages in many organisms, including humans, but tetraploid cells are rare in most normal human tissues.

Hypertension greatly increases the prevalence of these large, tetraploid cells in these vessels in humans, and Hixon and colleagues now show that the same is true of several animal models of hypertension. Following up on evidence that angiotensin is required for the effects of hypertension on the SMC cell cycle, these authors also demonstrate that expression of the protein kinase Akt1, a known mediator of angiotensin function, is elevated in capacitance arteries of hypertensive animals. Because the mitotic spindle checkpoint normally protects cells from undergoing extra rounds of DNA synthesis, Hixon et al. expressed Akt 1 in primary SMCs and tested the ability of these cells to activate the mitotic spindle checkpoint in response to microtubule-depolymerizing agents. They report that cyclin $\mathrm{B}$, a cell cycle regulator that participates in this checkpoint, is degraded prematurely in Akt1-expressing cells, despite the presence of these drugs. Furthermore, these cultures accumulate large, tetraploid cells even when they are not treated with spindle-damaging drugs, suggesting that increased Akt1 function is sufficient to produce this cellular phenotype in hypertensive or even in aging normotensive arteries.

\section{Golgi lipids regulate protein trafficking}

\section{(See article on pages 983-993)}

Cells rely on the Golgi apparatus to sort secreted proteins bound for different intracellular destinations. Membrane vesicles derived from this organelle may be targeted to the lysosome or the apical or basolateral surfaces of a polarized cell, or they may be held in the cyto- plasm awaiting a specific signal for exocytosis. Here, Choukroun and colleagues show that Golgi structure and function depend on local lipid metabolism. The phospholipase $\mathrm{CPLA}_{2}$ cleaves phospholipids to generate arachidonic acid, the precursor of many bioactive lipids, as well as lysophospholipids, which are thought to regulate membrane fusion during protein secretion. Choukroun et al. report that cPLA $_{2}$ associates with the cell's

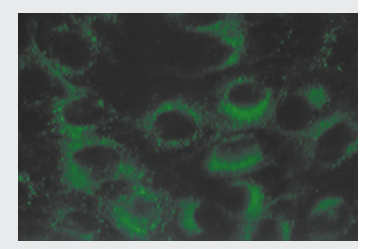

Golgi fraction, and they show that overexpression of the enzyme disrupts the organization of the Golgi apparatus and specifically blocks the trafficking of certain constitutively secreted proteins, while allowing other cargo proteins to be packaged normally into regulated secretory vesicles. The fragmentation of the Golgi apparatus seen in $\mathrm{CPLA}_{2}$-overexpressing cells is similar to a change that occurs during mitosis. The authors indicate that cPLA $_{2}$ activity rises during this period of the cell cycle, and they suggest that the accumulation of phospholipid metabolites in the Golgi membrane causes this organelle to vesiculate during cell division.

How streptococci go for the throat

\section{(See article on pages 995-1002)}

Pathogenic bacteria, like viruses, are accomplished molecular mimics, deploying surface molecules that interact with host-borne proteins in much the same way that endogenous host molecules do. Cywes and coworkers provide another clear example of this kind of adaptation in the ability of encapsulated strains of Streptococcus to bind epithelial cells of the pharynx and cause sore throats. Although no single component of the bacterium is required for binding to epithelial cells in culture, strains that lack the capsule fail to interact with the host cell receptor CD44 and are avirulent when presented in vivo. The bacterial capsule contains hyaluronic acid, the identical polysaccharide that is found in normal extracellular matrix and that interacts with CD44 on mammalian cells. Cywes et al. now show that epithelial cells of the pharynx express CD44 and that, when CD44 levels are suppressed using an antisense RNA, streptococci lose their capacity to colonize the throat. This interaction can also be blocked in vivo by treat-

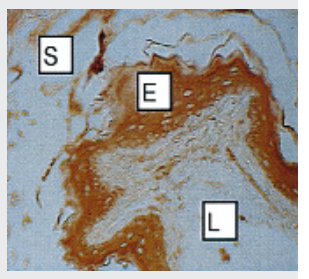
ing mice with exogenous hyaluronic acid or with antibodies to CD44, suggesting therapies that might treat a common source of misery and possibly other, life-threatening forms of streptococcal infection. 